\title{
Rapid Neutron Activation Analysis of Fissile Material Traces at the High Flux Isotope Reactor
}

\author{
David Glasgow ${ }^{1}$ and Justin Knowles ${ }^{2}$ \\ 1. Oak Ridge National Laboratory, Chemical Sciences Division, Oak Ridge, Tennessee, USA \\ 2. Oak Ridge National Laboratory, Nuclear Security and Isotope Technology Division, Oak Ridge, \\ Tennessee, USA
}

Neutron Activation Analysis (NAA) has a long history in forensic analysis of trace elements in materials [1] and exhibits many advantages for evidentiary forensics. Sample materials are introduced to the intense neutron field at the High Flux Isotope Reactor where elements within them become radioactive, giving up measurable radiation that is often unique to the elements themselves. The High Flux Isotope Reactor offers highly thermalized neutron irradiation of samples in two pneumatic transfer systems. Thermal neutron flux values for the PT-1 and PT-2 systems are $4.3 \mathrm{E}+14 \mathrm{n} \mathrm{cm}^{-2} \mathrm{~s}^{-1}$ and $4.0 \mathrm{E}+13 \mathrm{n} \mathrm{cm}^{-2} \mathrm{~s}^{-}$ 1 , respectively. The PT-2 facility connects to a sensitive neutron counter array (Figure 1) which is used to quantify neutrons given up by fission products created during the irradiation of traces of uranium. The measurement requires only a few minutes and yields a ${ }^{235} \mathrm{U}$ detection limit of about 20 picograms. Because NAA may be performed without sample dissolution or separation chemistries in many cases, the analysis of refractory solids is facilitated. NAA has been demonstrated by the National Institutes of Standards and Technology (NIST) to be capable of accuracy and precision commensurate with the demands of primary ratio methods such as Isotope Dilution Mass Spectrometry (IDMS) [2]. Furthermore, all of the sources of uncertainty are known and may be quantified [3]. The process of Neutron Activation Analysis may be modeled using the SCALE modeling suite [4] and detector response can be modeled using the Monte Carlo transport code MCNP [5], thereby facilitating virtual experiments in support of real ones.

The analysis of trace fissile material in automotive carpeting using NAA is presented here and may be indicative of application of NAA to post-interdiction forensic investigations. Because NAA can measure the trace element content in the carpet matrix itself, the degree to which soil and other substances may interfere with the uranium traces may be determined by monitoring elements such as $\mathrm{Mn}, \mathrm{Al}, \mathrm{Zn}$, and Th which are present in elevated amounts in soil but much lower in carpet. Triplicate samples of gray and black automotive carpeting were cut, cleaned and placed in cleaned polyethylene irradiation containers. The samples were cleaned by successive washings using $2 \%$ Micro $^{\circledR}-90$ detergent and ultra-pure water. The samples then were irradiated in the PT-1 facility for 60 seconds and counted on high-purity germanium detectors (HPGe) after decay times ranging from minutes to days. Trace elements were determined in groups determined by the product radioisotope half-life (Figure 2). Separately, flux monitors created by evaporation of inductively coupled plasma (ICP) calibration standards were irradiated and compared to the results of SCALE modeling using the flux shape characterization derived from the results of the MCNP model of HFIR and corrected by measurement of activation foils and wires. These experiments determined the matrix elements in the samples. Delayed neutron counting showed that the cleaned samples exhibited ${ }^{235} \mathrm{U}$ less than $20 \mathrm{pg}$, making the tracking of fissile material moves in automobiles possible using NAA of automobile carpet. This method may also determine the ${ }^{235} \mathrm{U} /{ }^{238} \mathrm{U}$ ratio nondestructively, thereby confirming the presence of nuclear material even when natural uranium from soil may be present. Both uranium isotopes may be determined without any sample dissolution or separation chemistry and the determination was blank-free because the materials were 
moved to clean containers prior to analysis. The rapid screening of large numbers of samples seems possible since the method is fast and requires only extremely simple preparation.

References:

[1] DS Karjala, California Law Review 59 (1971), p. 1011-1013, 1039-1071.

[2] RR Greenberg, Journal of Radioanalytical and Nuclear Chemistry 278 (2008), p. 238.

[3] J Kucera, P Bode, V Stepanek, Journal of Radioanalytical and Nuclear Chemistry 245 (2000), p. 115.

[4] I. C. Gauld et al, Nuclear Technology, 174 (2011), p. 169.

[5] MCNP6 Development Team, Los Alamos National Laboratory, LA-CP-13-00634 (2013).
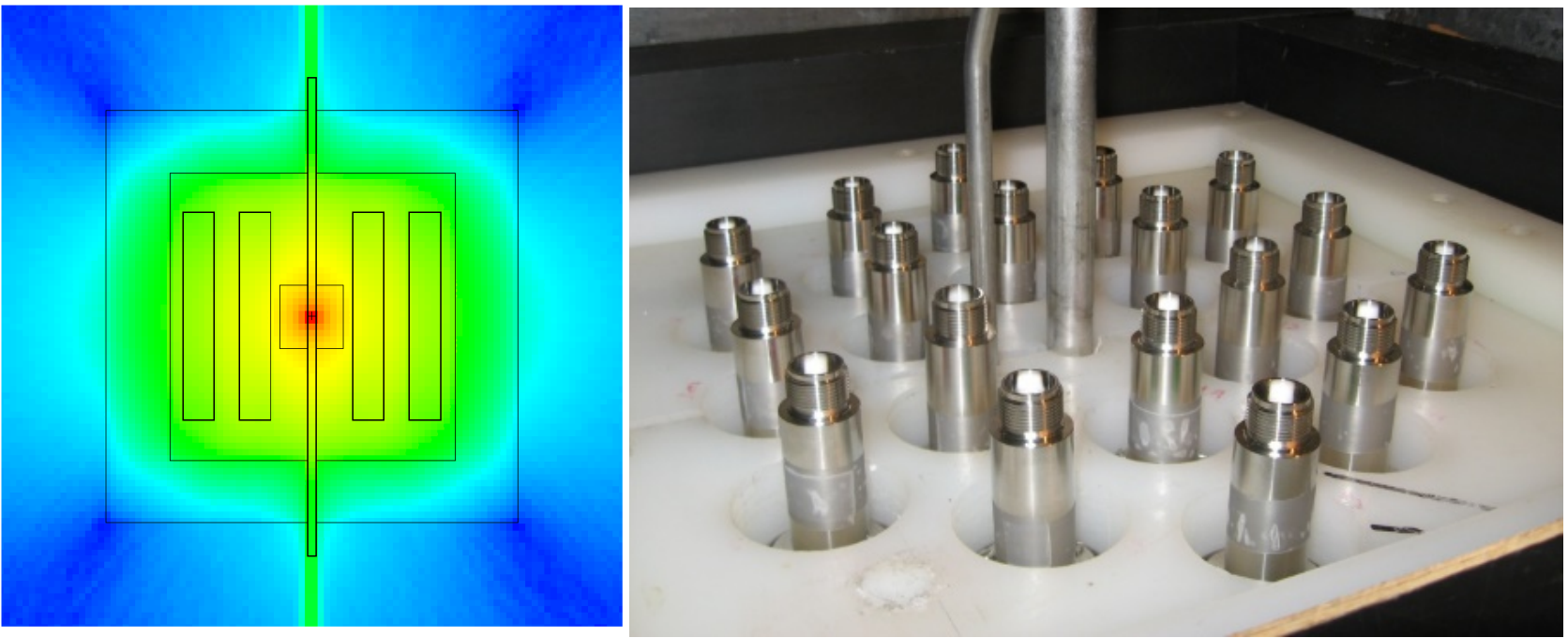

Figure 1. HFIR Delayed Neutron Detector Array MCNP Model (left) and Photo (right)

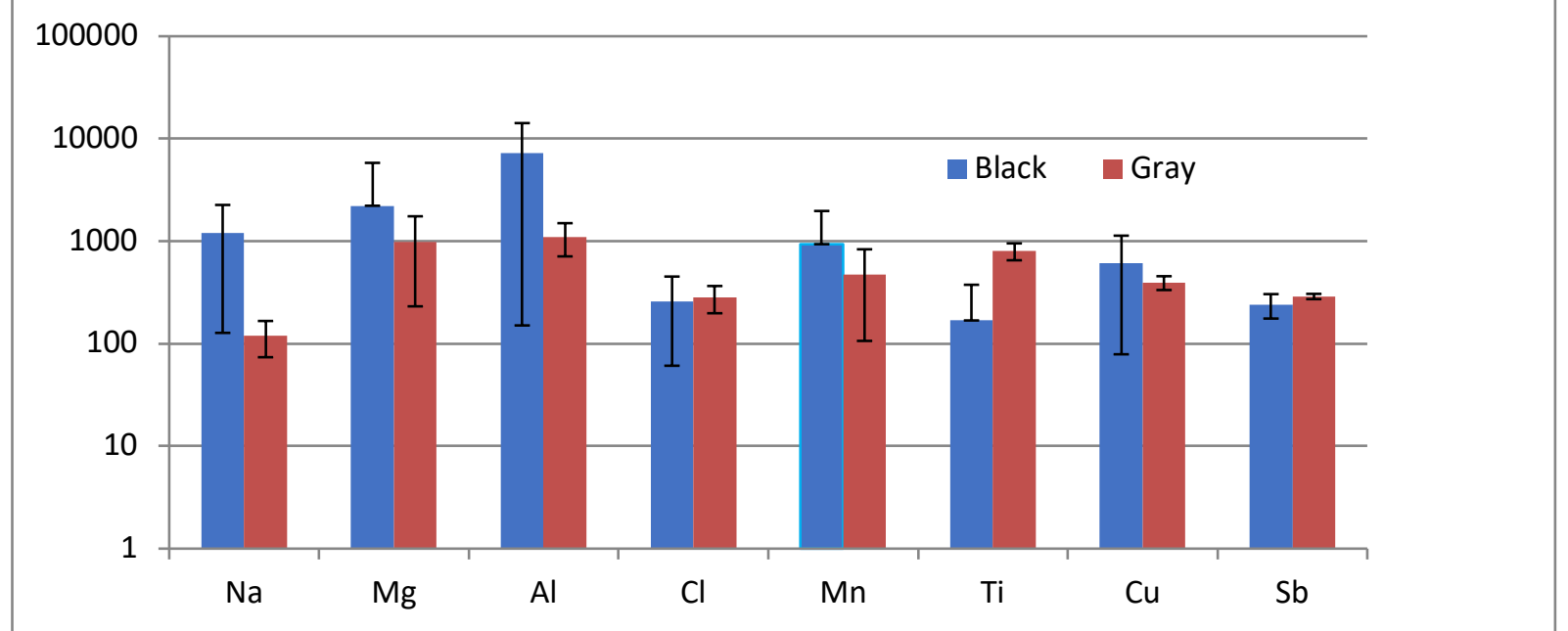

Figure 2. Matrix Elements Determined in Cleaned Gray and Black Carpet Samples. 